\title{
OPEN Micro computed tomography with and without contrast enhancement for the characterization of microcarriers in dry and wet state
}

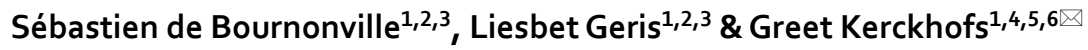

In the field of regenerative medicine, microcarriers are used as support matrix for the growth of adherent cells. They are increasingly recognised as promising biomaterials for large scale, costeffective cell expansion bioreactor processes. However, their individual morphologies can be highly heterogeneous which increases bioprocesses' variability. Additionally, only limited information is available on the microcarriers' 3D morphology and how it affects cell proliferation. Most imaging modalities do not provide sufficient 3D information or have a too limited field of view to appropriately study the 3D morphology. While microfocus X-ray computed tomography (microCT) could be appropriate, many microcarriers are hydrated before in-vitro use. This wet state makes them swell, changing considerably their morphology and making them indistinguishable from the culture solution in regular microCT images due to their physical density close to water. The use of contrast-enhanced microCT (CE-CT) has been recently reported for 3D imaging of soft materials. In this study, we selected a range of commercially available microcarrier types and used a combination of microCT and CE-CT for full 3D morphological characterization of large numbers of microcarriers, both in their dry and wet state. With in-house developed image processing and analysis tools, morphometrics of individual microcarriers were collected. Also, the morphology in wet state was assessed and related to accessible attachment surface area as a function of cell size. The morphological information on all microcarriers was collected in a publicly available database. This work provides a quantitative basis for optimization and modelling of microcarrier based cell expansion processes.

As the number of clinical trials for cell-based therapies is increasing, so does the need for robust and cost-effective large-scale biomanufacturing strategies. From 2014 to 2018 , the number of clinical trials initiated grew by $32 \%{ }^{1}$ and as of June 2019, more than 1000 clinical trials are underway, amongst which more than 600 for cell and gene-modified cell therapies ${ }^{2}$. Most of these therapies require the robust production of large number of cells, up to several billions, especially in the case of allogeneic therapies ${ }^{3,4}$. To overcome this challenge, an increasing number of innovative bioreactors are being developed, with more process monitoring and control features ${ }^{5}$. Over the last years, the use of microcarriers for the large scale expansion of adherent cells has been increasingly reported $^{6}$. The main advantage of such culture methods is the combination of a high 'attachment surface area to culture volume' ratio with the homogeneity of suspension cultures ${ }^{7}$. This makes it a promising solution for scalable and cost-efficient large scale biomanufacturing of adherent cells ${ }^{8-11}$. Table 1 gathers most of the commercially

\footnotetext{
${ }^{1}$ Division of Skeletal Tissue Engineering, KU Leuven, Leuven, Belgium. ${ }^{2}$ Biomechanics Section, Department of Mechanical Engineering, KU Leuven, Leuven, Belgium. ${ }^{3}$ Biomechanics Research Unit, ULiège, Liège, Belgium. "Biomechanics Lab, Institute of Mechanics, Materials and Civil Engineering, UCLouvain, Louvain-la-Neuve, Belgium. ${ }^{5}$ Department Materials Engineering, KU Leuven, Leuven, Belgium. ${ }^{6}$ Institute of Experimental and Clinical Research, UCLouvain, Woluwé-Saint-Lambert, Belgium. ${ }^{\varpi}$ email: greet.kerckhofs@ uclouvain.be
} 


\begin{tabular}{|c|c|c|c|c|c|c|}
\hline $\begin{array}{l}\text { Manufacturer \& } \\
\text { Microcarrier }\end{array}$ & $\begin{array}{l}\text { Number of microcarriers/g } \\
\text { dry weight }\end{array}$ & Density $(\mathrm{g} / \mathrm{ml})$ * & Particle size $(\mu \mathrm{m})^{*}$ & $\begin{array}{l}\text { Surface area }\left(\mathrm{cm}^{2} / \mathrm{g} \mathrm{dry}\right. \\
\text { weight })^{*}\end{array}$ & $\begin{array}{l}\text { Additional information on } \\
\text { morphology and matrix } \\
\text { composition }\end{array}$ & Reported use \\
\hline \multicolumn{7}{|c|}{ Microcarriers included in this study } \\
\hline GE Healthcare - Cytodex 1 & $4.3^{*} 10^{6}$ & 1.03 & 190 & 4400 & $\begin{array}{l}\text { Non-porous, swelling spher- } \\
\text { ical beads. Cross-linked } \\
\text { dextran and diethylaminoe- } \\
\text { thyl (DEAE) matrix }\end{array}$ & $16,28-32$ \\
\hline GE Healthcare - Cytodex 3 & $3.0^{*} 10^{6}$ & 1.04 & 175 & 2700 & $\begin{array}{l}\text { Non-porous, swelling } \\
\text { spherical beads. Dextran } \\
\text { matrix }\end{array}$ & $29,32-36$ \\
\hline $\begin{array}{l}\text { Percell Biolytica - Culti- } \\
\text { Spher S }\end{array}$ & $8.0^{*} 10^{5}$ & $1.03 \dagger$ & $255 \dagger$ & 7500 & $\begin{array}{l}\text { Macro-porous, swelling } \\
\text { beads. Gelatine matrix }\end{array}$ & $31,32,36-41$ \\
\hline $\begin{array}{l}\text { Corning - Dissolvable } \\
\text { Synthemax II }\end{array}$ & n.a & $1.025 \dagger$ & $250 \dagger$ & 5000 & $\begin{array}{l}\text { Non-porous swelling beads. } \\
\text { Polygalacturonic acid (PGA) } \\
\text { polymer chains cross-linked } \\
\text { via calcium ions and coated } \\
\text { with Synthemax II. Also } \\
\text { available untreated or with } \\
\text { Collagen coating }\end{array}$ & 14 \\
\hline $\begin{array}{l}\text { Corning-Polystyrene } \\
\text { untreated }\end{array}$ & n.a & 1.026 & $168.5 \dagger$ & 360 & $\begin{array}{l}\text { Non-porous, spherical } \\
\text { beads. Polystyrene matrix. } \\
\text { Also available with dif- } \\
\text { ferent coatings (Collagen, } \\
\text { positively charged, CellBind, } \\
\text { Synthemax II) }\end{array}$ & $10,42,43$ \\
\hline Pall SoloHill - Collagen & $4.6^{*} 10^{5}$ & $1.026 \dagger$ & $168.5 \dagger$ & 360 & $\begin{array}{l}\text { Non-porous, spherical } \\
\text { beads. Cross-linked poly- } \\
\text { styrene matrix coated with } \\
\text { collagen type 1 }\end{array}$ & $32,36,44,45$ \\
\hline Pall SoloHill - FACT 3 & $4.6^{*} 10^{5}$ & $1.026 \dagger$ & $168.5 \dagger$ & 360 & $\begin{array}{l}\text { Non-porous, spherical } \\
\text { beads. Cross-linked polysty- } \\
\text { rene matrix coated with col- } \\
\text { lagen type } 1 \text { and proprietary } \\
\text { surface modification }\end{array}$ & 32,44 \\
\hline Pall SoloHill - Hillex 2 & n.a & $1.120 \dagger$ & $180 \dagger$ & 515 & $\begin{array}{l}\text { Non-porous, spherical } \\
\text { beads. Polystyrene matrix } \\
\text { modified with cationic } \\
\text { amine }\end{array}$ & $32,44,45$ \\
\hline Pall SoloHill - Plastic & $4.6^{\star} 10^{5}$ & $1.026 \dagger$ & $168.5 \dagger$ & 360 & $\begin{array}{l}\text { Non-porous, spherical } \\
\text { beads. Styrene co-polymer } \\
\text { matrix }\end{array}$ & $32,45-48$ \\
\hline Pall SoloHill - Plastic Plus & $4.6^{*} 10^{5}$ & $1.026 \dagger$ & $168.5 \dagger$ & 360 & $\begin{array}{l}\text { Non-porous, spherical } \\
\text { beads. Styrene co-polymer } \\
\text { matrix with electric charge }\end{array}$ & $14,32,45,49$ \\
\hline Pall SoloHill - Star Plus & $4.6^{\star} 10^{5}$ & $1.026 \dagger$ & $168.5 \dagger$ & 360 & $\begin{array}{l}\text { Non-porous, spherical } \\
\text { beads. Cross-linked polysty- } \\
\text { rene matrix }\end{array}$ & 45 \\
\hline \multicolumn{7}{|c|}{ Microcarriers not included in this study } \\
\hline GE Healthcare - Cytopore 2 & $3.0^{*} 10^{6}$ & 1.03 & 230 & 11,000 & $\begin{array}{l}\text { Macro-porous, swelling non- } \\
\text { spherical beads. Cellulose } \\
\text { and DEAE matrix }\end{array}$ & 31,32 \\
\hline Pall SoloHill - ProNectin F & n.a & n.a & $120 \dagger$ & 480 & $\begin{array}{l}\text { Non-porous, spherical } \\
\text { beads. Polystyrene coated } \\
\text { with recombinant RGD- } \\
\text { containing protein }\end{array}$ & 32,50 \\
\hline Pall SoloHill - Glass & n.a & n.a & $168.5 \dagger$ & 360 & $\begin{array}{l}\text { Non-porous, spherical } \\
\text { beads. Cross-linked poly- } \\
\text { styrene coated with High } \\
\text { silica glass }\end{array}$ & \\
\hline MP Biomedical - RapidCell & n.a & $1.03 \dagger$ & $180 \dagger$ & 325 & $\begin{array}{l}\text { Non-porous, spherical } \\
\text { beads. Glass matrix }\end{array}$ & 51 \\
\hline $\begin{array}{l}\text { Tosoh Biosciences - Tosoh } \\
65 \text { PR }\end{array}$ & n.a & n.a & 65 & 4200 & $\begin{array}{l}\text { Non-porous, spherical } \\
\text { beads. Hydroxylated meth- } \\
\text { acrylate with Tresyl ligand } \\
\text { derivatized and Protamine } \\
\text { sulphate surface treatment }\end{array}$ & 30 \\
\hline $\begin{array}{l}\text { Tosoh Biosciences - Tosoh } \\
10 \mathrm{PR}\end{array}$ & n.a & n.a & 10 & 9000 & $\begin{array}{l}\text { Non-porous, spherical } \\
\text { beads. Hydroxylated meth- } \\
\text { acrylate with Tresyl ligand } \\
\text { derivatized and Protamine } \\
\text { sulphate surface treatment }\end{array}$ & 30 \\
\hline Nunc - Biosilon & n.a & 1.05 & 190 & 225 & $\begin{array}{l}\text { Non-porous, spherical } \\
\text { beads. Polystyrene matrix }\end{array}$ & 16 \\
\hline Continued & & & & & & \\
\hline
\end{tabular}




\begin{tabular}{|l|l|l|l|l|l|}
\hline $\begin{array}{l}\text { Manufacturer \& } \\
\text { Microcarrier }\end{array}$ & $\begin{array}{l}\text { Number of microcarriers/g } \\
\text { dry weight }\end{array}$ & Density $(\mathbf{g} / \mathbf{m l}) *$ & Particle size $(\boldsymbol{\mu m})^{*}$ & $\begin{array}{l}\text { Surface area }\left(\mathbf{c m}^{2} / \mathbf{g} \text { dry }\right. \\
\text { weight) }\end{array}$ & $\begin{array}{l}\text { Additional information on } \\
\text { morphology and matrix } \\
\text { composition }\end{array}$ \\
\hline Whatman - DE-53 & n.a & 1.1 & L: 130, D: 35 & 6800 & $\begin{array}{l}\text { Non-porous cylindrical } \\
\text { microcarriers. Cellulose } \\
\text { matrix with DEAE surface } \\
\text { treatment. Also available } \\
\text { with lower charge density } \\
\text { (DE-52) or different surface } \\
\text { treatments (QA-52, CM52) }\end{array}$ \\
\hline New Brunswick - Fibra-Cel & n.a & n.a & $\begin{array}{l}\text { Macro-porous, disk-shape } \\
\text { microcarriers. Polyester } \\
\text { non-woven fibre and poly- } \\
\text { propylene matrix }\end{array}$ \\
\hline
\end{tabular}

Table 1. Commonly used commercially available microcarriers for cell expansion processes. ${ }^{\star}$ Metrics in fully hydrated state (where applicable). $\uparrow$ Average calculated from the range provided by the manufacturer. n.a.: Not available.

available microcarriers, reported for cell expansion bioprocesses. However, challenges remain to make the use of this technology an industrial reality.

Harvesting cells from the microcarriers with a high efficiency while maintaining their quality, is one of them, hence making it one of the most critical steps of the process ${ }^{12}$. Nowadays, new types of dissolvable and external stimulus-responsive microcarriers (i.e. thermo-, electro- or light-responsive) are being developed in the hope of overcoming this limitation ${ }^{13-21}$. An additional challenge is the high morphological heterogeneity of most microcarriers within one production batch, and, more importantly, the lack of quantitative information on their morphological properties, despite being critical process parameters ${ }^{22-24}$. The heterogeneity introduces external variability in the system and hampers stochastic analyses of bioprocesses focused on cell-related variability. Additionally, it prevents the study and optimization of cell attachment behaviour on new substrate morphologies.

Furthermore, many of the microcarriers developed in the field need to be hydrated in a preparation solution in order to swell before being used in process (cf. Table 1). This process changes drastically the morphology of the microcarrier. Although the substrate morphology is of critical importance in the bioprocesses, accurate data on the 3D morphology of microcarriers in their wet state is hardly available. For most microcarriers, the available information is insufficient to quantify and analyse their morphological variability. At best, some microcarrier size and surface area ranges are given by the manufacturers.

While microscope or SEM-based imaging modalities are commonly used for visual evaluation of cell growth on microcarriers, these techniques are inappropriate for precise full 3D morphological analyses of microcarriers. Indeed, such analyses would require a precise $3 \mathrm{D}$ imaging modality that can image the external and internal microstructure of a representative number of microcarriers at the same time, both in their dry and wet state. As it provides a wide field of view to image a large amount of microcarriers at the same time, and since it is a nondestructive imaging technique allowing full 3D imaging of materials, microfocus X-ray computed tomography (microCT) is appropriate for imaging microcarriers in their dry state. However, standard absorption microCT is inappropriate to visualise microcarriers in their wet state, because of their density being close to water. Contrastenhanced microCT (CE-CT) has been recently reported as an innovative approach for 3D visualisation of soft materials in their wet state ${ }^{25,26}$. CE-CT consists of using high atomic number chemical compounds that bind to specific soft materials in order to increase their X-ray attenuation coefficient, also referred to as contrastenhancing staining agents (CESAs) ${ }^{27}$.

In this study, we used (i) microCT to image microcarriers in their dry state and (ii) CE-CT to image the microcarriers in their wet state. Together with in-house developed dedicated image processing algorithms, they enabled high throughput, high-resolution visualisation of single microcarriers and their morphological characterization. By imaging microcarriers both in their dry and wet state with the same imaging modality, quantification of the microcarrier swelling and of the attachment surface area were performed. With this study, we provided a publicly available, representative database of $3 \mathrm{D}$ morphometrics for the most used microcarriers in adherent cell expansion processes, both in their dry and wet state. This database provides a basis for the optimisation of attachment conditions in microcarrier-based cell cultures, depending on cell size. Additionally, this can provide future studies with more insights in microcarrier morphology-related process variability and enables bioprocess stochastic modelling.

\section{Results}

3D visualisation of microcarriers in dry and wet state. Figure 1 shows typical 2D cross-sections from all types of microcarriers, for some both in dry and wet state. The cross section of the wet CultiSpher S sample with no contrast agent shows the poor contrast between the microcarriers and PBS and highlights the need to use CESAs for the imaging of such biomaterials in wet state. Swelling due to hydration of the CultiSpher S, Cytodex 1, Cytodex 3 and Synthemax can be observed by comparing the dry and wet state images of these microcarriers. All 2D slices of swelling microcarriers show a general increase in microcarrier size in wet state. Swelling of CultiSpher S microcarriers is also highlighted in Movie 1.

As previously reported, Zr-Kgg POM binds electrostatically to positively charged or collagen containing structures $^{25}$. Figure 1 shows the effective contrast enhancement thanks to the binding of the CESA to the gelatine matrix of CultiSpher S, the positively charged diethylaminoethyl groups of Cytodex 1 and the collagen layer of 


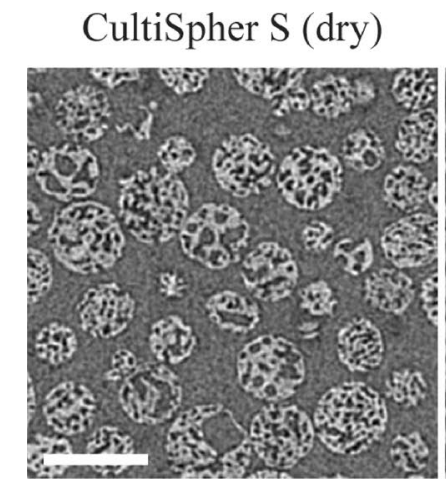

Cytodex 3 (dry)
CultiSpher S (wet)

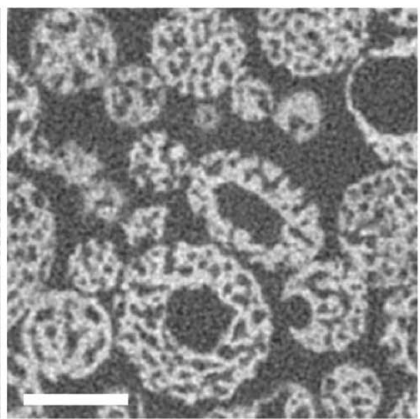

Cytodex 3 (wet)
Cytodex 1 (dry)

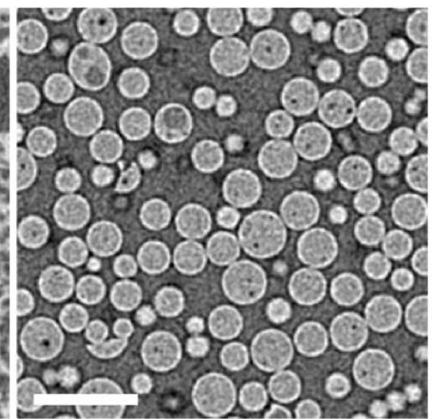

Synthemax II (dry)
Cytodex 1 (wet)

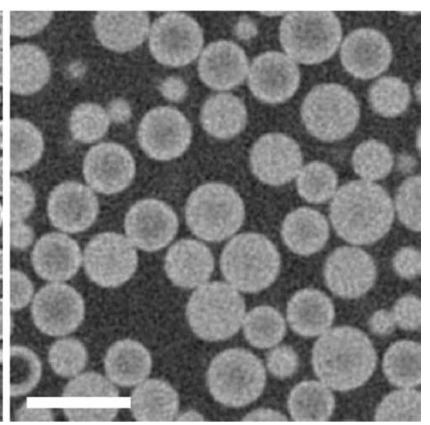

Synthemax II (wet)
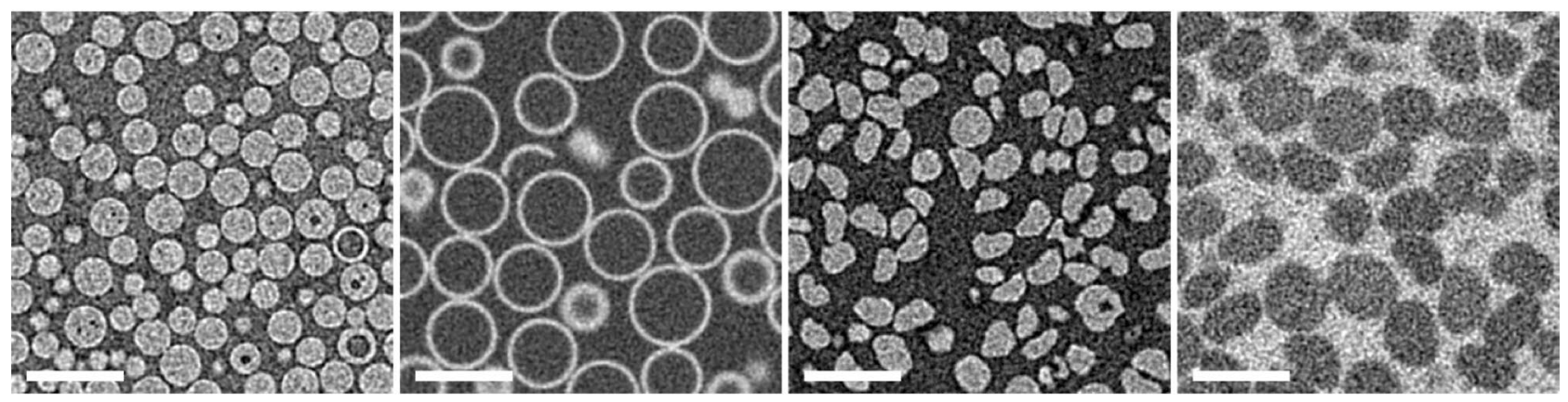

Collagen (dry)

Corning (dry)

Fact 3 (dry)

Hillex 2 (dry)
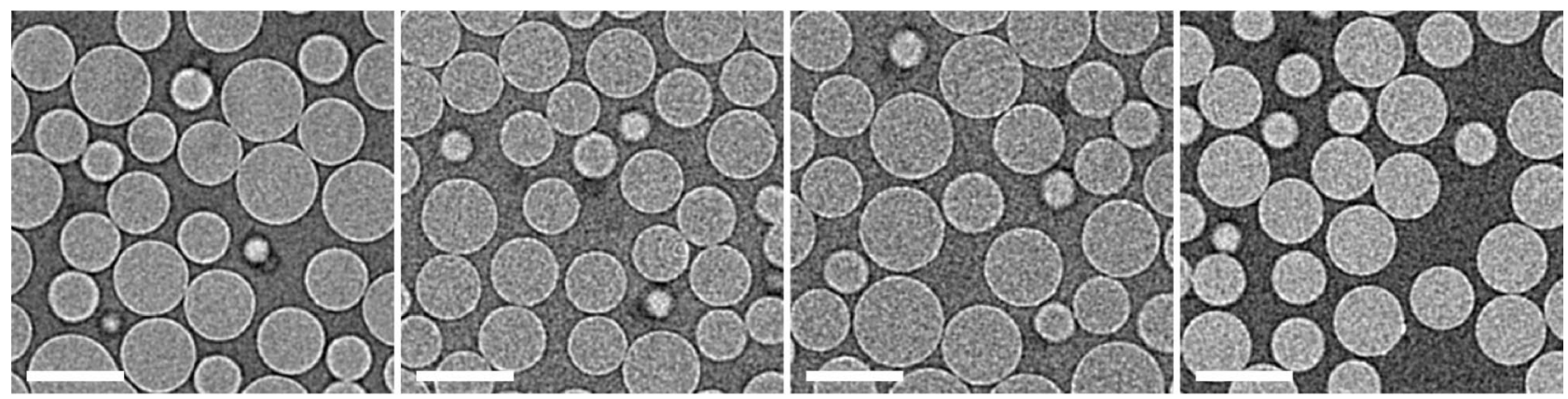

Plastic (dry)

Plastic $+($ dry $)$

Star + (dry)
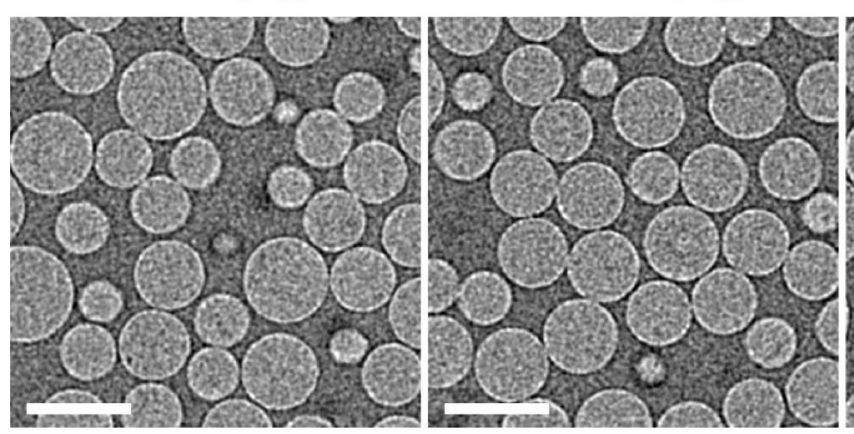

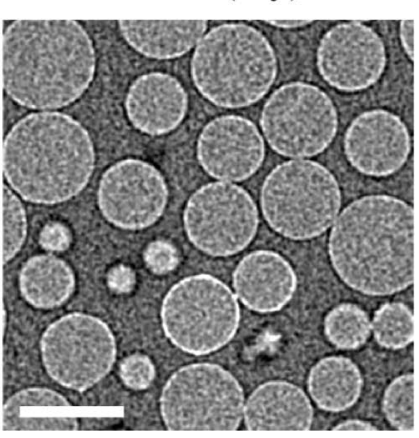

CultiSpher S

(wet w/o CESA)

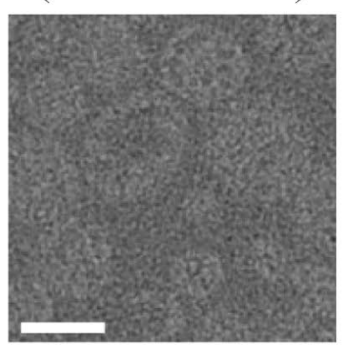

Figure 1. Typical 2D micro-CT and CE-CT cross-sections from all microcarriers types in dry and some in wet state. The bottom right shows a microCT cross-section of CultiSpher S in wet state, without CESA staining. Scale bars $=200 \mu \mathrm{m}$.

Cytodex 3. The effective repulsion of Hexabrix by the negative charges of Synthemax II microcarriers is also clear (Fig ure1), making them appear darker than the suspension solution.

Morphological characterization of the microcarriers. The morphometrics of all microcarrier types, for each microcarrier in the dataset, were gathered in a publicly available Mendeley Data repository accessible here: http://dx.doi.org/10.17632/rf6hsw3f2d. $1^{52}$. 

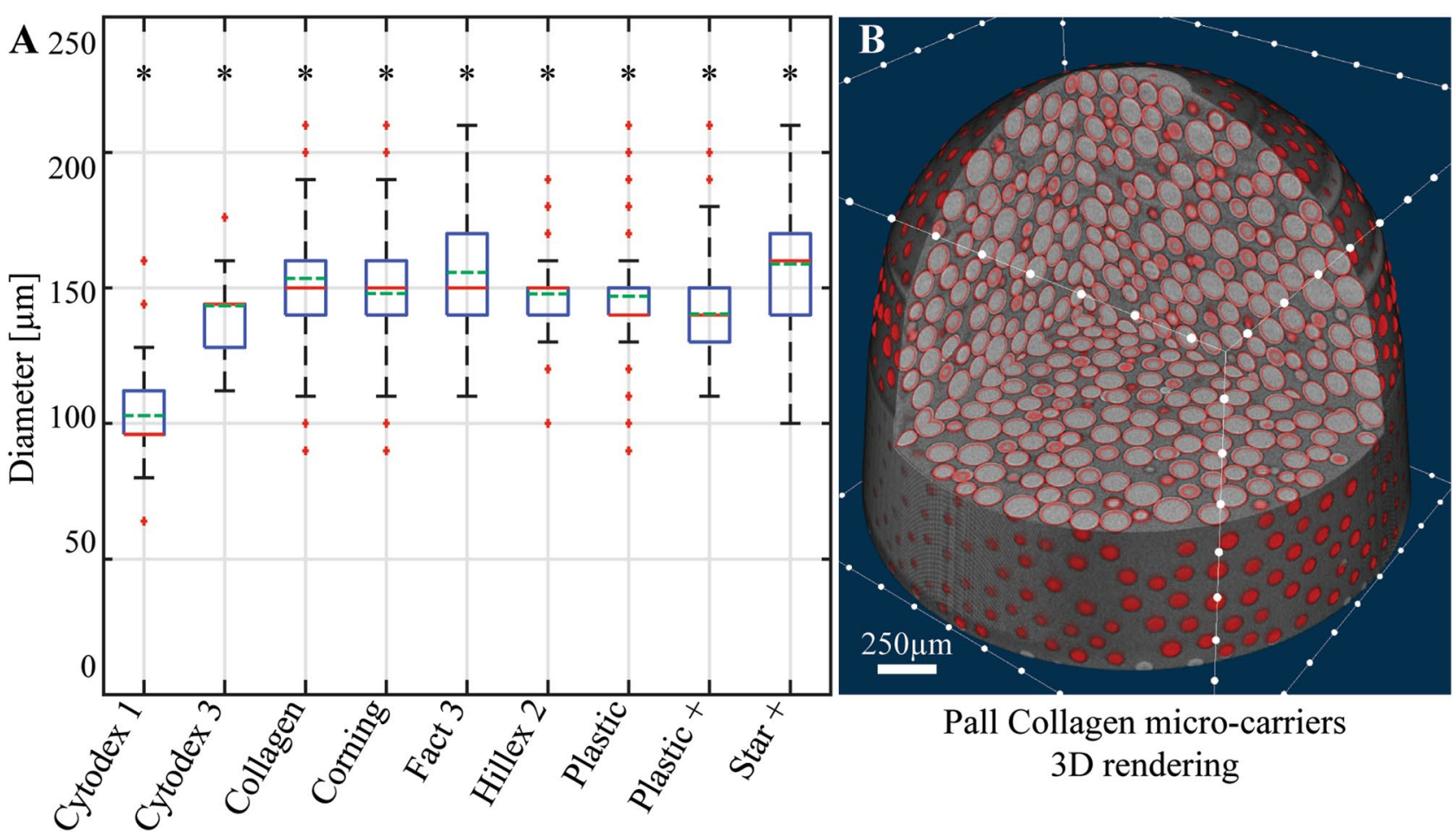

Figure 2. Results of the Hough transform on spherical microcarriers in wet state. (A) Calculated spherical microcarrier diameter distribution, shown as box plots. The asterisks indicate the rejection of the hypothesis that the data comes from a standard normal distribution (5\% significance level). (B) 3D rendering of the Pall Collagen microcarrier dataset (grayscales) overlapped with binary spherical shells (red) calculated from the identified sphere centres and radii detected by the Hough transform.

Supplementary Figs. 2 and 3, respectively, show normalized histogram distributions of the equivalent diameters and surface areas for all the microcarrier types, and give an overview of the variability in their morphology.

Spherical microcarriers. Figure 2A shows boxplots of the calculated microcarrier diameters for the spherical beads, in wet state when applicable. The radii, surface areas and microcarrier volumes can be found in the repository. None of these morphometrics followed a symmetrical distribution.

Figure 2B and Movie 2 show a 3D rendering of a sample of Pall Collagen microcarriers (grey), along with the fitted spheres from the Hough transform (red). This provides a qualitative assessment of the performance of the algorithm. Good fit was observed between all modelled sphere volumes and their original datasets, for all spherical microcarrier types.

Non-spherical microcarriers.

1. Validation of the segmentation algorithm

The results of the automatic segmentation algorithm are illustrated in Movie 3 and a still of that Movie is shown on Fig. 3. Figures $3 \mathrm{~A}$ and $3 \mathrm{~B}$ show a $3 \mathrm{D}$ renderings of validation dataset 1, overlapped with the watershed contour from the segmentation, respectively. Figures $3 \mathrm{C}$ and $3 \mathrm{D}$ show the CultiSpher S sample in wet state before segmentation and after segmentation, respectively, where all individual microcarriers were assigned a random grayscale value between 30 and 255 to visually assess the quality of the segmentation.

The manual segmentation of the validation datasets led to the identification and isolation of 249 individual microcarriers, while the automatic segmentation algorithm led to the segmentation of 211 microcarriers, thus showing a $15 \%$ underestimation of the number of microcarriers with the automatic segmentation algorithm. The comparison of the distribution of the morphometrics calculated from the two segmentation methods is summarized in Table 2, as validation for the segmentation algorithm. This table shows that the automatic segmentation algorithm leads to similar results than the manual segmentation. However, the pore volume and porosity were significantly different. A significant difference between both segmentation methods was also observed on the Principal axis 1 length, although with a p-value was close to the significance threshold (0.042).

2. Morphometrics

Figure 4 shows the results of the calculation of the convex hull, the open pores and the closed pores on an individual CultiSpher S microcarrier. 


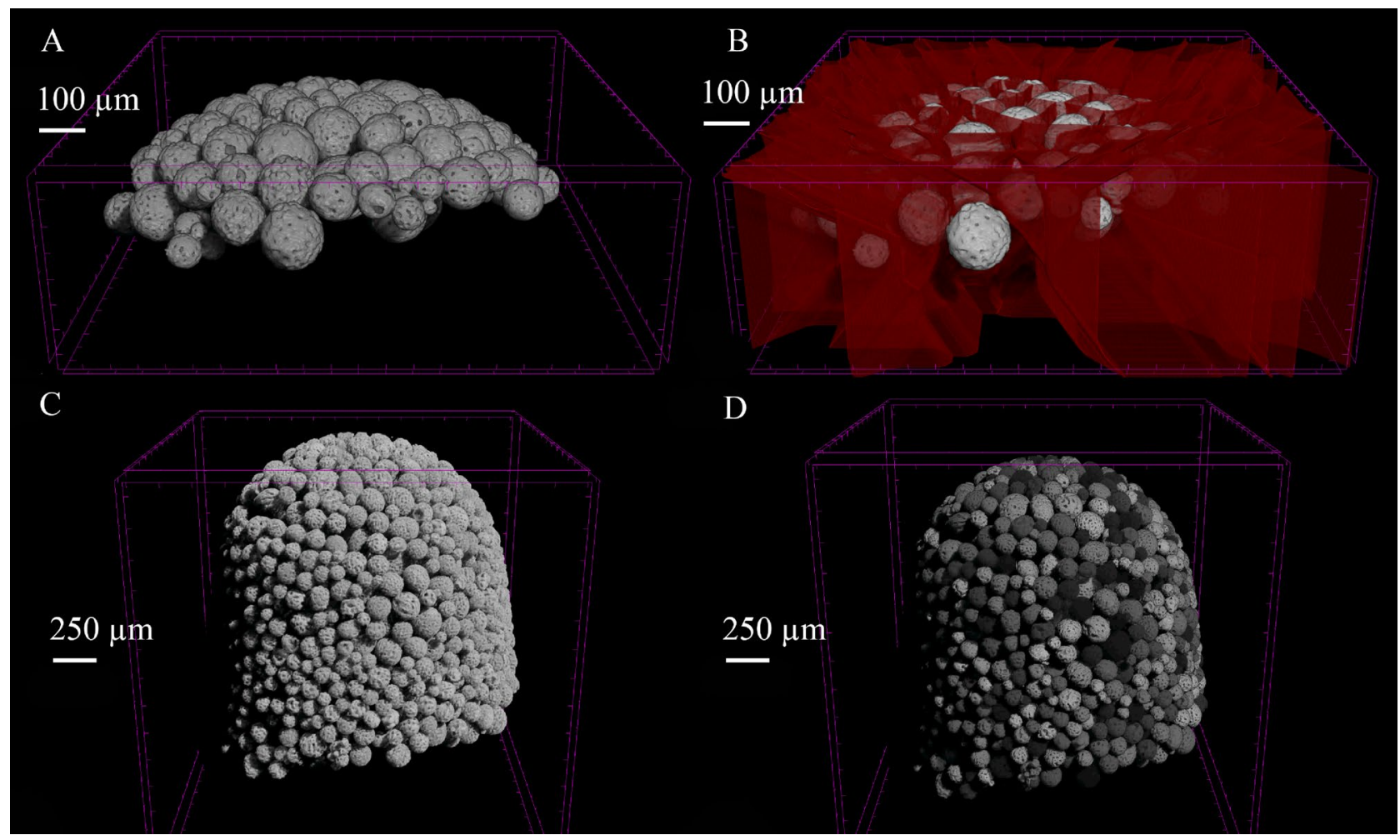

Figure 3. Still of Movie 3. 3D visualisation of the segmentation algorithm results on CultiSpher S microcarriers. (A) Binarized validation sample. (B) Binarized validation sample (white) overlapped with watershed segmentation contours (red). (C) Binarized CultiSpher S sample before segmentation. (D) Binarized CultiSpher $\mathrm{S}$ sample after segmentation, individual microcarriers were all given a random grayscale value.

\begin{tabular}{|c|c|c|c|c|c|c|c|c|c|c|c|c|}
\hline \multirow[b]{2}{*}{ Morphometric } & \multicolumn{5}{|c|}{ Manual segmentation } & \multicolumn{2}{|l|}{ T-test } & \multicolumn{5}{|c|}{ Automatic segmentation } \\
\hline & Avg & Std & Q25 & Median & Q75 & $\alpha=5 \%$ & $p$ & Avg & Std & Q25 & Median & Q75 \\
\hline Equivalent Diameter $[\mu \mathrm{m}]$ & 199.18 & 72.28 & 146.83 & 211.64 & 243.79 & & 0.144 & 208.52 & 63.16 & 153.73 & 212.09 & 245.86 \\
\hline Principal Axis 1 Length $[\mu \mathrm{m}]$ & 190.76 & 73.28 & 137.09 & 197.81 & 232.40 & * & 0.042 & 204.00 & 64.25 & 157.88 & 200.69 & 234.80 \\
\hline Principal Axis 2 Length $[\mu \mathrm{m}]$ & 177.76 & 64.20 & 129.98 & 190.05 & 219.66 & & 0.134 & 186.21 & 54.86 & 138.10 & 190.77 & 220.46 \\
\hline Principal Axis 3 Length $[\mu \mathrm{m}]$ & 168.16 & 59.98 & 125.89 & 180.48 & 201.72 & & 0.305 & 173.64 & 53.27 & 133.20 & 177.02 & 201.06 \\
\hline Convex surface area $\left[\mu \mathrm{m}^{\wedge} 2\right]$ & $1.41 \mathrm{E} 5$ & $9.04 \mathrm{E} 4$ & $6.71 \mathrm{E} 4$ & $1.40 \mathrm{E} 5$ & $1.88 \mathrm{E} 5$ & & 0.253 & $1.50 \mathrm{E} 5$ & $8.94 \mathrm{E} 4$ & $7.54 \mathrm{E} 4$ & $1.41 \mathrm{E} 5$ & $1.91 \mathrm{E} 5$ \\
\hline Bead volume $\left[\mu \mathrm{m}^{\wedge} 3\right]$ & $4.43 \mathrm{E} 6$ & $4.07 \mathrm{E} 6$ & $1.28 \mathrm{E} 6$ & $3.91 \mathrm{E} 6$ & $6.00 \mathrm{E} 6$ & & 0.677 & $4.28 \mathrm{E} 6$ & $3.83 \mathrm{E} 6$ & $1.39 \mathrm{E} 6$ & $3.54 \mathrm{E} 6$ & $5.44 \mathrm{E} 6$ \\
\hline Convex hull volume $\left[\mu \mathrm{m}^{\wedge} 3\right]$ & $5.73 \mathrm{E} 6$ & $5.33 \mathrm{E} 6$ & $1.66 \mathrm{E} 6$ & $4.96 \mathrm{E} 6$ & $7.59 \mathrm{E} 6$ & & 0.477 & $6.09 \mathrm{E} 6$ & $5.57 \mathrm{E} 6$ & $1.90 \mathrm{E} 6$ & $5.00 \mathrm{E} 6$ & $7.78 \mathrm{E} 6$ \\
\hline Open pore volume $\left[\mu \mathrm{m}^{\wedge} 3\right]$ & $1.24 \mathrm{E} 6$ & $1.29 \mathrm{E} 6$ & $3.69 \mathrm{E} 5$ & $9.52 \mathrm{E} 5$ & $1.58 \mathrm{E} 6$ & * & $1.75 \mathrm{E}-4$ & $1.79 \mathrm{E} 6$ & $1.80 \mathrm{E} 6$ & $7.39 \mathrm{E} 5$ & $1.40 \mathrm{E} 6$ & $2.14 \mathrm{E} 6$ \\
\hline Closed pore volume $\left[\mu \mathrm{m}^{\wedge} 3\right]$ & $5.56 \mathrm{E} 4$ & $1.28 \mathrm{E} 5$ & $1.01 \mathrm{E} 3$ & $1.12 \mathrm{E} 4$ & $4.79 \mathrm{E} 4$ & * & 0.004 & $2.46 \mathrm{E} 4$ & $9.86 \mathrm{E} 4$ & $3.68 \mathrm{E} 2$ & $3.62 \mathrm{E} 3$ & $1.31 \mathrm{E} 4$ \\
\hline Porosity [/] & 0.23 & 0.05 & 0.20 & 0.22 & 0.26 & * & $8.31 \mathrm{E}-30$ & 0.30 & 0.07 & 0.26 & 0.29 & 0.33 \\
\hline
\end{tabular}

Table 2. Validation results of the automatic segmentation algorithm against manual segmentation, using distributions of morphometrics (column 1). For each morphometric (rows), the average value, the standard deviation, the quantile $25 \%$, the median and the quantile $75 \%$ are calculated from the manually segmented microcarriers (columns 2-6) and the automatically segmented microcarriers (columns 9-13). Both methods are statistically compared for each resulting morphometric using a Student's t-test, $n=249$ (manual) and $\mathrm{n}=211$ (automatic) (columns $7-8)$. ${ }^{\star}$ indicates statistical significance $(\alpha=0.05)$.

For CultiSpher S, an increase of 51.64\% in equivalent diameter (Fig. 5A) and of $147.56 \%$ in convex surface area (Fig. 5C) were observed due to swelling. For Synthemax II, swelling led to an increase of $58.77 \%$ in equivalent diameter (Suppl. Figure 4A) and of $151.82 \%$ in convex surface area (Suppl. Figure 4C). The three principal axes of the microcarriers indicated that the CultiSpher $\mathrm{S}$ microcarriers have a generally more spherical shape (Fig. 5B) than the Synthemax II ones (Suppl. Figure 4B), both in dry and wet state. This was confirmed visually (3D renderings of the microcarriers, Fig. 5E-G and Suppl. Figures4E-G). Varying the probing radius of the alpha shapes allowed visualisation of the accessible microcarrier surface area for a given cell size (Fig. 5E-G and Suppl. Figures4E-G). The available surface area was also plotted as a function of the cell size (Fig. 5D and Suppl. Figures4D). For small cell sizes $(\sim 4 \mu \mathrm{m})$, the median available surface area for cell attachment increased 


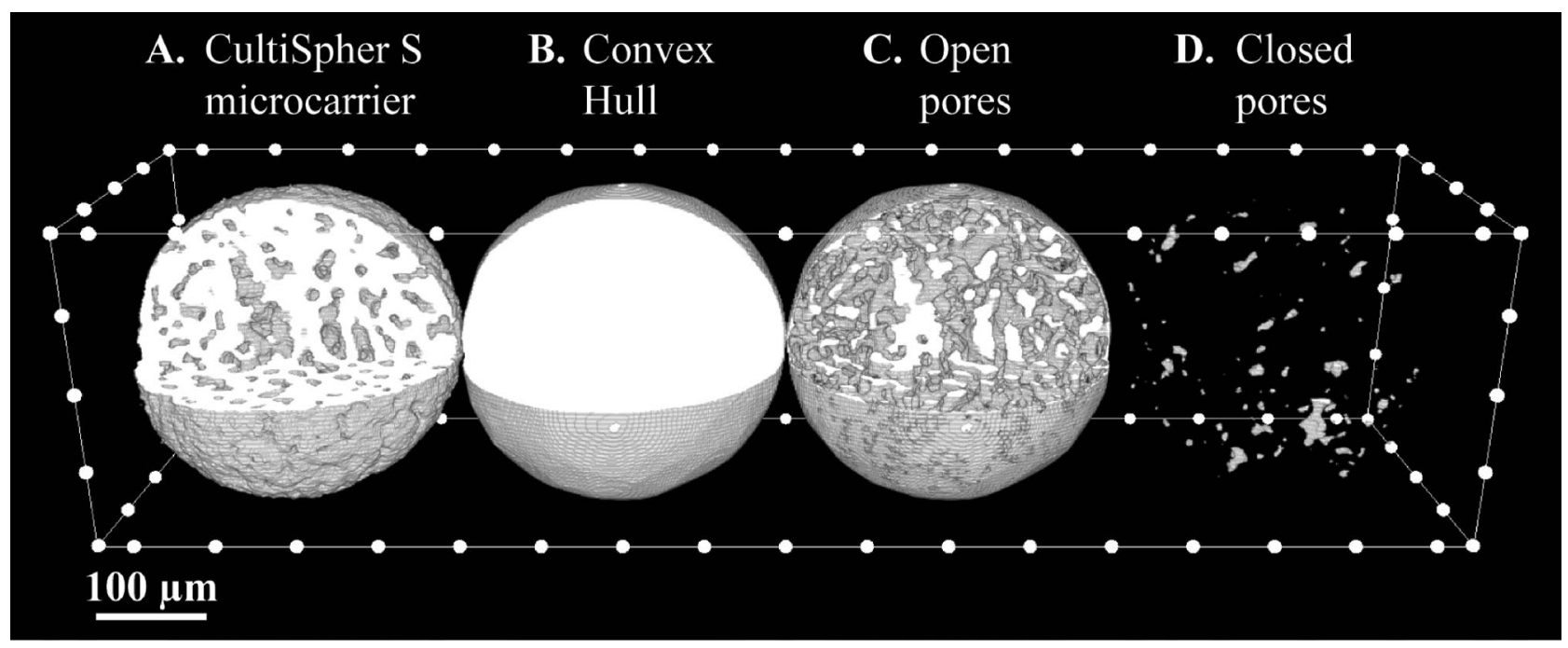

Figure 4:. 3D visualisation of an individual CultiSpher S. From left to right: the microcarrier (A), the calculated convex hull (B), open pores (C) and closed pores (D).

by a factor 2.60 for CultiSpher S and 1.08 for Synthemax II, compared to the convex surface area, accessible to bigger cells $(\geq 50 \mu \mathrm{m})$.

The distribution of the surface area to volume ratio (Fig. 6) shows that the non-spherical microcarriers, Cytodex 1 and Synthemax II, had the highest surface area to volume ratio.

Comparison to the microcarrier specifications from the manufacturers. Supplementary Table 3 compares the morphometrics calculated in this study (for the swelling microcarriers) to the morphological information specified by the manufacturers (accessible attachment surface area per dry weight and estimated microcarrier size). Similar values to the manufacturers were found for the non-swelling microcarriers. Lower values were found for our calculations for the morphometrics of the swelling microcarriers, especially for the surface area per dry weight and the size of the Cytodex microcarriers.

\section{Discussion}

3D visualisation of microcarriers in dry and wet state. This study showed that, by using the proper CESA for each biomaterial, the combined use of microCT and CE-CT is a highly suitable technique to image microcarriers both in their dry and wet state (Fig. 1). The generated datasets provided full 3D imaging of high numbers of microcarriers per sample, which was suitable to analyse the morphometrics from each individual microcarrier. The morphometrics were gathered in a database and their histogram distributions show the large variability in morphological properties of microcarriers in one batch. They form the basis for accurate stochastic modelling of microcarrier-based bioprocesses. To the best of our knowledge, this study is the first report in the field showing full 3D morphometrics of individual microcarriers. For swelling microcarriers, a change in the morphometric distribution was observed after swelling, indicating that microcarriers are affected unequally by the swelling process; this induces even increased variability.

Validation of the segmentation algorithm. There was good agreement between the morphometrics determined using our in-house developed automatic segmentation algorithm and the ones determined using manual segmentation. However, significant differences were observed for morphometrics related to porosity (porosity, open pore volume, closed pore volume), which can be explained by the sensitivity of those morphometrics to the accuracy of the segmentation algorithm. A small over-segmentation of one microcarrier will introduce high concavity in the morphology of that microcarrier, which will directly influence those parameters. A significant difference between the two methods was also observed for the first principal axis of the microcarrier, which can also be explained by the sensitivity of this parameter to over-segmentation.

The underestimation of the number of microcarriers likely comes from the high number of small microcarriers, as observed on the manually segmented dataset (black arrows on Supplementary Figs. 5 and 6). Such small microcarriers are less easily detected by the automatic segmentation algorithm, and therefore induce a bias in the statistics. Additionally, their presence highlights the morphological heterogeneity of CultiSpher S microcarriers.

In general, the results show that the segmentation algorithm performed well, with limitations regarding porosity and a small over-estimation of the first principal axis. However, manual segmentations are prone to errors and both intra- and inter-operator variations. Thus, further manual segmentations should be performed to increase the accuracy of the ground truth dataset and decrease operator bias.

Morphometrics of microcarriers. Spherical microcarriers (polystyrene and dextran matrices) showed narrower equivalent diameter and surface area distributions than the non-spherical ones (Suppl. Figures 2 and 

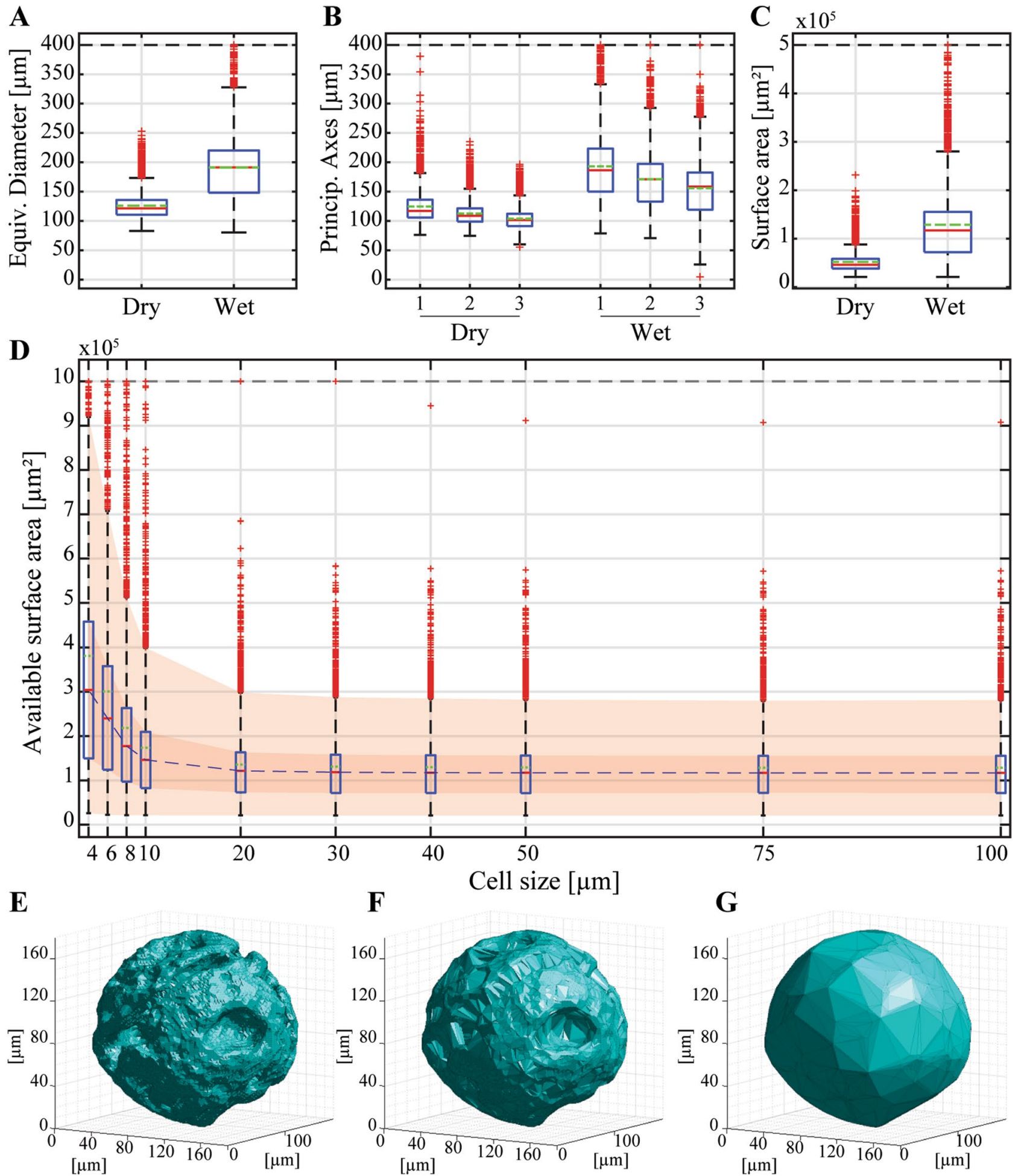

Figure 5. Morphometrics of individual CultiSpher $S$ microcarriers in dry $(n=2820)$ and wet $(n=2958)$ state. (A) Boxplot distributions of the equivalent diameter. (B) Boxplot distributions of the 3 principal axes. The axes are numbered from 1 to 3 in each state. (C) Boxplot distributions of the convex surface area. (D) Boxplot distributions of the available surface area for cell attachment as a function of the cell size. (E-G) $3 \mathrm{D}$ representations of the calculated alpha shapes for a probing radius (三 cell size) of (E) $4,(\mathbf{F}) 12$ and (G) $+\infty \mu \mathrm{m}$. The latter corresponds to the convex hull of the microcarrier.

3), which indicates a more controlled manufacturing process. Narrow morphometric distributions are more suitable for standard use in bioprocesses, as they provide more homogeneous cell culture conditions. In addition 


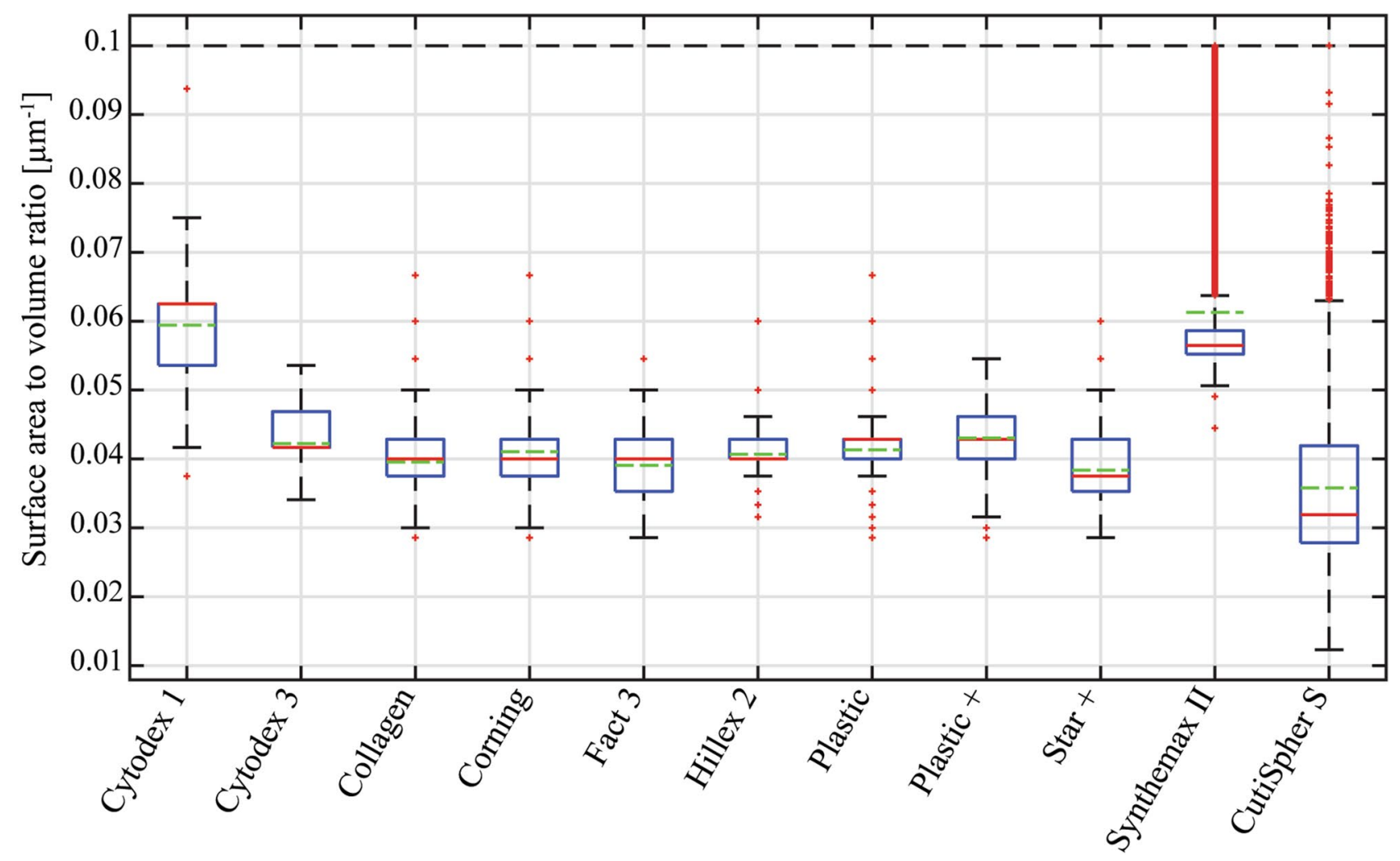

Figure 6. Boxplot distributions of surface area to volume ratio for all microcarrier types, in wet state.

to providing a less homogeneous culture environment, attention should be paid to high numbers of small microcarriers in the distributions (i.e. for CultiSpher S and Synthemax II, Supplementary Fig. 2). Indeed, while these microcarriers increase the total available surface area for attachment, it is likely that the attachment behaviour of cells on these microcarriers with higher surface curvature will be different ${ }^{53}$, which again generally increases the heterogeneity in the process.

The Alpha-Shape method allowed to relate cell size (三 probing radius) with accessible attachment surface area in the case of porous (CultiSpher S) and concave (Synthemax II) microcarriers. The increase in accessible surface area for cell attachment was more pronounced for CultiSpher $\mathrm{S}$ than for Synthemax II, due to their macro-pores. However, the additional attachment surface area provided by these pores can only be significantly accessed by small cells $(10 \mu \mathrm{m}$ and smaller, cf. Fig. 5D). These results suggest that the throat size of the CultiSpher S pores is rather small, and that the concave and porous morphologies of Synthemax II and CultiSpher S microcarriers only play a significant role for small cells. Thus, cell size should be accounted for when calculating the seeding density for bioprocessing.

The differences observed between the morphometrics and the data provided by manufacturers for wet microcarriers may be due to their swelling. Swelling generally broadens the histograms of morphometrics, which makes their comparison to average values less relevant. Additionally, the estimates of number of microcarriers per dry weight is highly sensitive to artefacts in the segmentation algorithm, which might also explain these discrepancies. Further studies should be performed on the swelling behaviour and the quantification of the number of microcarriers per dry weight.

\section{Conclusions}

In this study, we showed that CE-CT could be used as a novel technique to image soft biomaterials, such as microcarriers, in their wet state. Combining with standard microCT allowed to assess the swelling of the microcarriers. Both microCT and CE-CT enabled the precise, full 3D imaging and morphological characterization of different types, and high and representative numbers, of microcarriers. Dedicated 3D image processing and analysis (partly developed in-house) allowed quantification of the morphometrics of individual microcarriers (collected in an online database), and the assessment of the swelling behaviour of some microcarrier types. The representative morphometric distributions of all microcarriers form the basis for optimisation of cell culture conditions and bioprocess modelling, in which accurate variability studies can now be conducted. With this study, we have shown that CE-CT can be recognised as innovative quality control tool for the morphology of microcarriers in wet state, and that it can be used for the optimization of new microcarrier-based bioprocesses. In the future, the development of a cell-specific CESA should be considered, to enable the visualisation of single cells on microcarriers, and understand better their attachment behaviour. 


\section{Methods}

Microcarriers. Several commercially available and commonly reported microcarriers for use in large-scale cell expansion experiments were selected for this study and are shown in Table 1.

Swelling and staining of the microcarriers. For the CultiSpher S, Cytodex 1 and Cytodex 3 microcarriers, we used a polyoxometalate (POM) as CESA for CE-CT. A $3.5 \%(35 \mathrm{mg} / \mathrm{ml})$ staining solution of a Zirconiumsubstituted (Zr-Kgg) POM was prepared in $\mathrm{PBS}^{54}$. The microcarriers were incubated in the solution over night to allow them to swell and have the CESA staining them. The staining time was optimized for the microcarriers by evaluating for full diffusion of the CESA within the matrix.

The Synthemax II microcarriers were hydrated in PBS $(0.15 \mathrm{~mL}$ per milligram of microcarriers) for $10 \mathrm{~min}$, according to the manufacturer's protocol. Because the observed repulsion of those microcarriers to negatively charged CESAs, such as the POM, we used Hexabrix as a CESA for these microcarriers. Hexabrix contains a negatively charged ioxaglate ${ }^{55,56}$. Hexabrix was added to reach a concentration of $10 \%$ ( $1 \mathrm{ml}$ Hexabrix in $10 \mathrm{ml}$ PBS). This CESA provided a 'negative' staining of the microcarriers, i.e. the surrounding liquid was contrasted, but the microcarriers were not, as the CESA did not stain them.

MicroCT and CE-CT image acquisition. A sample of 6-10 mg was taken for each microcarrier type. All microcarrier samples were first imaged in Eppendorf Tubes in dry state, and then in wet state, for the swelling microcarriers (cf. Table 1). All samples were imaged using a Phoenix NanoTom M (GE Measurement and Control Solutions, Germany) with a voxel size of $2 \mu \mathrm{m}, 1200$ images per rotation, in fast scan mode (average of 1 and Skip of 0 ), with a diamond-coated tungsten target in modus 0 , using a collimator and no filter. For the samples in dry state, a voltage of $50 \mathrm{kV}$, a current of $160 \mu \mathrm{A}$, and an exposure time of 750 ms were used. For the CultiSpher $\mathrm{S}$, Cytodex 1 and Cytodex 3 samples in wet state, a voltage of $70 \mathrm{kV}$, with a $120 \mu \mathrm{A}$ current and a 500 ms exposure time were used. Finally, for the Corning dissolvable Synthemax II (further referred to as Synthemax II) samples in wet state, a voltage of $60 \mathrm{kV}$, with a current of $140 \mu \mathrm{A}$ and an exposure time of $1000 \mathrm{~ms}$ were used.

All 3D renderings of CT and CE-CT datasets presented here were generated using the CTvox software v3.1.1 r1191 (64-bit) (https://www.bruker.com/products/microtomography/micro-ct-software/3dsuite.html, Bruker Micro-CT, Kontich, Belgium).

Image processing. All datasets were first processed in the CTAn software v1.17 (https://www.bruker.com/ products/microtomography/micro-ct-software/3dsuite.html, Bruker Micro-CT, Kontich, Belgium) to remove the Eppendorf Tubes from the images by drawing circular regions of interest (ROIs). Then the datasets were processed with Matlab 2017b (MathWorks, MA, USA) as described below. Different protocols were applied for the spherical and non-spherical microcarriers.

Image analysis of spherical microcarriers. The datasets of the spherical microcarriers (cf. Table 1-rows 1 to 4) were first resized prior to image analysis, for computational efficiency. Then, a 3D spherical shape recognition algorithm (Hough transform) was implemented, using an algorithm previously developed by Xie et al. ${ }^{57}$, with a minimum and maximum radius, $R_{\min }$ and $R_{\max }$ respectively. From the Hough transform, the radius and centre of the spherical microcarriers could be calculated. From the radius of each of the single microcarriers, i.e. $R_{i}$, the approximate surface area and volume were calculated as $4 \pi R_{i}^{2}$ and $\frac{4}{3} \pi R_{i}^{3}$, respectively. The minimum and maximum radius were chosen according to information provided by the manufacturer. The radii for all spherical microcarrier datasets can be found in Supplementary Table 1 . To visually assess the fit between the results from the Hough transform and the datasets, 3D binary masks were built for each sample, containing binary shells (thickness $10 \mu \mathrm{m}$ ) whose centres and radii were taken from the results of the Hough transform.

Image analysis of non-spherical microcarriers. For the non-spherical microcarriers (cf. Table 1 - rows 5 to 11), first a segmentation algorithm was applied on the datasets to isolate single beads. Then, individual microcarriers were analysed and morphometrics were calculated. All analyses were performed in Matlab. The segmentation algorithm consisted of the following steps:

Step 1 Binarization using the automatic multilevel Otsu's method ${ }^{58}$ with two thresholds, as the images consisted of two background peaks and one microcarrier peak in the histogram. For the wet Synthemax II microcarriers, the grayscale values between the two thresholds were selected as foreground (microcarrier) because of the negative staining, while for the CultiSpher S microcarriers, the grayscale values above the highest threshold were selected as foreground because of the positive staining (see histogram on Supplementary Figure S1).

Step 2 From the binary images, all objects (defined as connected components with a 3D connectivity of 18) with a volume smaller than $V_{\text {small }}$ were removed to filter out noise, using the function bwareaopen, creating MASK1.

Step 3 Using the function $b w$ dist on MASK1, the Euclidean distance transform of the background was computed. This distance transform was then binarized using a threshold radius $R_{p}$ (referring to half of the pore thickness) to define MASK2, containing all the voxels situated at a distance greater than $R_{p}$ in the image, hence disconnecting the pores from the background.

Step 4 A binary morphological opening of the MASK2 was performed with the function imopen, using a spherical structuring element of radius $R_{o p}$, to disconnect the remaining pores from the background, resulting in MASK3. 
Step 5 By using the function imclearborder on MASK3, the remaining pore objects were isolated and were cleared out of the mask by combining it with the inverse of the isolated pore objects, in a Boolean operation AND.

The resulting binary object was then dilated using a binary morphological dilation with a spherical structuring element of radius $R_{p}$, allowing to dilate the edge of the background object back to the microcarrier boundary, creating MASK4.

Step 6 The inverse of MASK4 is a binarization of touching microcarriers, with closed pores. Thus, a distance transform combined with a watershed segmentation, as described by Fernand Meyer ${ }^{59}$, was used to segment the different microcarriers (function watershed). To decrease segmentation artefacts, the distance transform was filtered with a gaussian filter (function imgaussfilt 3 with standard deviation 7).

Intermediate results of this algorithm, applied to CultiSpher S microcarriers in wet state, are shown in Supplementary Fig. 1. The parameter values used for every dataset are listed in Supplementary Table 2. After segmentation, all microcarriers in each dataset were individually analysed in $3 \mathrm{D}$ to compute the morphometrics and their distributions per sample.

As the Synthemax II microcarriers are not porous, Steps 3 and 5 were skipped for these datasets. Also, an intermediate closing (with radius $R_{c l}$ ) and removal of small black volumes were added to remove background noise emerging from the negative staining. For Synthemax II, the function regionprops 3 was used to compute the microcarrier volume, equivalent diameter (assuming a sphere of the same volume) and the three principal axis lengths, calculated as the length of the major axes of the ellipsoid that have the same normalized second central moments as the volume of the microcarrier.

For the CultiSpher S datasets, both in dry and wet state, regionprops 3 was first used to compute the convex hull image of the microcarrier. Let $I_{i}$ and $I_{c o n v, i}$ respectively be the 3D binary images of microcarrier $i$ (Fig. 4A) and its convex hull (Fig. 4B). By inverting $I_{i}$ and clearing all objects connected to the image borders, the closed pores $I_{c l, i}$, of the microcarrier can be found (Fig. 4D). From these, the compact microcarrier (filled closed pores) was defined as $I_{f i l l, i}=I_{i} \bigvee I_{c l, i}$ and the open pores of the microcarrier can be calculated as $I_{o p, i}=I_{c o n v, i} \bigwedge\left[\neg I_{f i l l, i}\right]$ (Fig. 4C). Summing up the true voxels of these variables allows the calculation of the microcarrier volume $V_{i}$, the convex hull volume $V_{c o n v, i}$, the open pore volume $V_{o p, i}$ and the closed pore volume $V_{c l, i}$. Porosity of an individual microcarrier was calculated as $P_{i}=1-\frac{V_{i}}{V_{\text {conv }}}$. Using regionprops 3 on $I_{c o n v, i}$ allowed calculation of the equivalent diameter, the three principal axis lengths and the convex surface area.

For calculating the surface area of both Synthemax II and CultiSpher S microcarriers, an alpha-shape method was implemented, as described by Edelsbrunner et al. ${ }^{60}$. Varying the probing radius allowed to relate the available surface area for cell attachment to the cell size. An infinite probing radius returned the convex surface area of the microcarrier, which was confirmed by comparing this technique to the Crofton's formula ${ }^{61}$ (implemented in regionprops 3 ) applied on $I_{c o n v, i}$. Thus, the $3 \mathrm{D}$ coordinates of the foreground pixels of $I_{i}$ were used as set point input to build the alpha shapes with probing radii ranging between $[2,3,4,10,15,20,25,38,50,+\infty]$ voxels, therefore relating to cell sizes ranging between $[4,6,8,20,30,40,50,76,100,+\infty] \mu \mathrm{m}$.

To validate our segmentation algorithm, a manual segmentation of all individual microcarriers was performed on two smaller samples of CultiSpher S microcarriers. The individual segmentations were performed using the ROI drawing tool of CTAn (Bruker Micro-CT, Kontich, Belgium), each time saving single microcarrier datasets and removing them from the sample dataset. The automatic segmentation algorithm was also on both datasets. Then, individual morphometrics were calculated on the individual microcarriers, segmented from both techniques, and results were compared. To increase statistical power, both CultiSpher S datasets were combined in each group.

Surface area per dry weight. From the calculated morphometrics, the attachment surface area per dry weight of microcarrier can be estimated. This parameter is highly relevant to the bioprocess and makes a link between the characterization and the function of the microcarrier. Additionally, it is one the few experimentally determined metrics that can be compared to the morphological information provided by the manufacturers. For all microcarriers that do not swell, this parameter can be calculated as $\frac{\widetilde{S}}{\widetilde{V}}$ where $\tilde{S}$ and $\widetilde{V}$ are the median surface area and median volume of the microcarrier dataset (respectively) and $V_{d}^{* d}$ is the density of that microcarrier. For the swelling microcarriers, this parameter was estimated as the median surface area of the microcarriers $S$ multiplied by the number of microcarriers per dry weight (Table 1). However, since this number was not available for the Synthemax II, it was estimated from the ratio of the number of microcarriers imaged in the dry sample, over the sample weight.

Statistical analyses. To quantify significant differences between morphometrics of CultiSpher $S$ calculated from the two different segmentation methods, a paired t-test was performed in Matlab. To test the null hypotheses of standard normal distribution of morphometrics, a one-sample Kolmogorov-Smirnov test was used. In all test cases, $\mathrm{p}=0.05$ was considered significant.

The multiple boxplots shown throughout the paper should be interpreted as follows: the green bars indicate the average value, the red bars show the median values, the blue ones show the $25 \%$ and $75 \%$ quantiles, the vertical dotted black lines show the whiskers (minimum and maximum values without outliers) and the red crosses indicate outliers. Morphometrics were considered outliers if their value was greater than $q_{3}+1.5 *\left(q_{3}-q_{1}\right)$ or less than $q_{1}-1.5 *\left(q_{3}-q_{1}\right)$, where $q_{1}$ and $q_{3}$ are respectively the 25 th and 75 th percentiles of the morphometric. For clarity of the figures, extreme data limits are used on the boxplots. These are shown as horizontal dotted black lines. Outside these limits, outliers are displayed as clipped at the limit. 
Received: 8 June 2020; Accepted: 21 December 2020

Published online: 02 February 2021

\section{References}

1. Alliance for Regenerative Medicine. Clinical trials in Europe: recent trends in ATMP development. (2019).

2. Lambert, J. Getting Ready: Act now for access to ATMPs in Europe. Getting Ready: Act now for access to ATMPs in Europe (2019).

3. Simaria, A. S. et al. Allogeneic cell therapy bioprocess economics and optimization: single-use cell expansion technologies. Biotechnol. Bioeng. 111, 69-83 (2014).

4. Abraham, E., Ahmadian, B. B., Holderness, K., Levinson, Y. \& McAfee, E. Platforms for Manufacturing Allogeneic, Autologous and iPSC Cell Therapy Products: An Industry Perspective. in New Bioprocessing Strategies : Development and Manufacturing of Recombinant Antibodies and Proteins (eds. Kiss, B., Gottschalk, U. \& Pohlscheidt, M.) 165, 323-350 (Springer, New York, 2018).

5. de Bournonville, S. et al. towards self-regulated bioprocessing: a compact benchtop bioreactor system for monitored and controlled 3D cell and tissue culture. Biotechnol. J. 14, e1800545 (2019).

6. Lambrechts, T. et al. Large-scale mesenchymal stem/stromal cell expansion: a visualization tool for bioprocess comparison. Tissue Eng. Part B. Rev. 22, 485-498 (2016).

7. Badenes, S. M. et al. Microcarrier culture systems for stem cell manufacturing. in Stem Cell Manufacturing 77-104 (Elsevier Inc., 2016). https://doi.org/10.1016/B978-0-444-63265-4.00004-2

8. Panchalingam, K. M., Jung, S., Rosenberg, L. \& Behie, L. A. Bioprocessing strategies for the large-scale production of human mesenchymal stem cells: a review mesenchymal stem/stromal cells - an update. Stem Cell Research and Therapy 6, 225 (2015).

9. Mizukami, A. et al. Technologies for large-scale umbilical cord-derived MSC expansion: experimental performance and cost of goods analysis. Biochem. Eng. J. 135, 36-48 (2018).

10. Sousa, M. F. Q. et al. Production of oncolytic adenovirus and human mesenchymal stem cells in a single-use, vertical-wheel bioreactor system: Impact of bioreactor design on performance of microcarrier-based cell culture processes. Biotechnol. Prog. 31, 1600-1612 (2015).

11. Rowley, J., Abraham, E., Campbell, A., Brandwein, H. \& Oh, S. Meeting lot-size challenges of manufacturing adherent cells for therapy. Bioprocess Int. 10, 16-22 (2012).

12. Derakhti, S., Safiabadi-Tali, S. H., Amoabediny, G. \& Sheikhpour, M. Attachment and detachment strategies in microcarrier-based cell culture technology: a comprehensive review. Mater. Sci. Eng. C 103, (2019).

13. Yang, H. S., Jeon, O., Bhang, S. H., Lee, S.-H. \& Kim, B.-S. Suspension culture of mammalian cells using thermosensitive microcarrier that allows cell detachment without proteolytic enzyme treatment. Cell Transplant. 19, 1123-1132 (2010).

14. Rodrigues, A. L. et al. Dissolvable microcarriers allow scalable expansion and harvesting of human induced pluripotent stem cells under xeno-free conditions. Biotechnol. J. 14, 1800461 (2019).

15. Nguyen, L. T. B. et al. Development of thermo-responsive polycaprolactone macrocarriers conjugated with Poly(N-isopropyl acrylamide) for cell culture. Sci. Rep. 9, 1-11 (2019).

16. Çetinkaya, G., Kahraman, A. S., Gumusderelioglu, M., Arat, S. \& Onur, M. A. Derivation, characterization and expansion of fetal chondrocytes on different microcarriers. Cytotechnology 63, 633-643 (2011).

17. Tamura, A., Kobayashi, J., Yamato, M. \& Okano, T. Thermally responsive microcarriers with optimal poly(N-isopropylacrylamide) grafted density for facilitating cell adhesion/detachment in suspension culture. Acta Biomater. 8, 3904-3913 (2012).

18. Park, B. R., Nabae, Y., Surapati, M., Hayakawa, T. \& Kakimoto, M. A. Poly(N-isopropylacrylamide)-modified silica beads with hyperbranched polysiloxysilane for three-dimensional cell cultivation. Polym. J. 45, 210-215 (2013).

19. Song, K. et al. In vitro culture and harvest of BMMSCs on the surface of a novel thermosensitive glass microcarrier. Mater. Sci. Eng. C 58, 324-330 (2016).

20. Yuan, X., Tsai, A. C., Farrance, I., Rowley, J. A. \& Ma, T. Aggregation of culture expanded human mesenchymal stem cells in microcarrier-based bioreactor. Biochem. Eng. J. 131, 39-46 (2018).

21. Higuchi, A. et al. External stimulus-responsive biomaterials designed for the culture and differentiation of ES, IPS, and adult stem cells. Prog. Polym. Sci. 39, 1585-1613 (2014).

22. Sart, S. \& Agathos, S. N. Large-scale expansion and differentiation of mesenchymal stem cells in microcarrier-based stirred bioreactors. in Methods in Molecular Biology 1502, 87-102 (Humana Press Inc., 2016).

23. Sart, S., Agathos, S. N. \& Li, Y. Engineering stem cell fate with biochemical and biomechanical properties of microcarriers. Biotechnol. Prog. 29, 1354-1366 (2013).

24. Tavassoli, H. et al. Large-scale production of stem cells utilizing microcarriers: a biomaterials engineering perspective from academic research to commercialized products. Biomaterials 181, 333-346 (2018).

25. Kerckhofs, G. et al. Simultaneous three-dimensional visualization of mineralized and soft skeletal tissues by a novel microCT contrast agent with polyoxometalate structure. Biomaterials 159, 1-12 (2018).

26. De Bournonville, S., Vangrunderbeeck, S. \& Kerckhofs, G. Contrast-enhanced microCT for virtual 3D anatomical pathology of biological tissues: a literature review. Contrast Media Mol. Imaging 2019, (2019).

27. de Bournonville, S. et al. Exploring polyoxometalates as non-destructive staining agents for contrast-enhanced microfocus computed tomography of biological tissues. Acta Biomater. 105, 253-262 (2020).

28. Schop, D. et al. Expansion of human mesenchymal stromal cells on microcarriers: growth and metabolism. J. Tissue Eng. Regen. Med. 4, 131-140 (2010).

29. Goh, T. K. P. et al. Microcarrier culture for efficient expansion and osteogenic differentiation of human fetal mesenchymal stem cells. Biores. Open Access 2, 84-97 (2013).

30. Chen, A. K. L., Chen, X., Choo, A. B. H., Reuveny, S. \& Oh, S. K. W. Critical microcarrier properties affecting the expansion of undifferentiated human embryonic stem cells. Stem Cell Res. 7, 97-111 (2011).

31. Ikonomou, L., Drugmand, J.-C., Bastin, G., Schneider, Y.-J. \& Agathos, S. N. Microcarrier Culture of Lepidopteran Cell Lines: Implications for Growth and Recombinant Protein Production. https://doi.org/10.1021/bp0255107 (2002).

32. Timmins, N. E. et al. Closed system isolation and scalable expansion of human placental mesenchymal stem cells. Biotechnol. Bioeng. 109, 1817-1826 (2012).

33. Hewitt, C. J. et al. Expansion of human mesenchymal stem cells on microcarriers. Biotechnol. Lett. 33, 2325-2335 (2011).

34. Chen, A. K. L., Chew, Y. K., Tan, H. Y., Reuveny, S. \& Oh, S. K. W. Increasing efficiency of human mesenchymal stromal cell culture by optimization of microcarrier concentration and design of medium feed. Cytotherapy 17, 163-173 (2015).

35. Caruso, S. R. et al. Growth and functional harvesting of human mesenchymal stromal cells cultured on a microcarrier-based system. Biotechnol. Prog. 30, 889-895 (2014).

36. Alfred, R. et al. Efficient suspension bioreactor expansion of murine embryonic stem cells on microcarriers in serum-free medium. Biotechnol. Prog. 27, 811-823 (2011).

37. Eibes, G. et al. Maximizing the ex vivo expansion of human mesenchymal stem cells using a microcarrier-based stirred culture system. J. Biotechnol. 146, 194-197 (2010). 
38. Santos, F. D. et al. Toward a clinical-grade expansion of mesenchymal stem cells from human sources: a microcarrier-based culture system under xeno-free conditions. Tissue Eng. C Methods 17, 1201-1210 (2011).

39. Gupta, P., Geris, L., Luyten, F. P. \& Papantoniou, I. An integrated bioprocess for the expansion and chondrogenic priming of human periosteum-derived progenitor cells in suspension bioreactors. Biotechnol. J. 13, 1700087 (2018).

40. Yuan, Y., Kallos, M. S., Hunter, C. \& Sen, A. Improved expansion of human bone marrow-derived mesenchymal stem cells in microcarrier-based suspension culture. J. Tissue Eng. Regen. Med. 8, 210-225 (2014).

41. Chen, M. et al. A modular approach to the engineering of a centimeter-sized bone tissue construct with human amniotic mesenchymal stem cells-laden microcarriers. Biomaterials https://doi.org/10.1016/j.biomaterials.2011.06.054 (2011).

42. Hervy, M. et al. Long term expansion of bone marrow-derived hmscs on novel synthetic microcarriers in xeno-free, defined conditions. PLoS ONE 9, e92120 (2014).

43. Cunha, B. et al. Exploring continuous and integrated strategies for the up- and downstream processing of human mesenchymal stem cells. J. Biotechnol. 213, 97-108 (2015).

44. Szczypka, M., Splan, D., Woolls, H. \& Brandwein, H. Single-Use Bioreactors and Microcarriers Scalable Technology for Cell-Based Therapies. 54 BioProcess International 12, (2014).

45. Splan, D. et al. Microcarrier-Based Xeno-Free Expansion of Human Mesenchymal Stromal Cells in a Single-Use Stirred-Tank Bioreactor. (2018).

46. dos Santos, F. et al. A xenogeneic-free bioreactor system for the clinical-scale expansion of human mesenchymal stem/stromal cells. Biotechnol. Bioeng. 111, 1116-1127 (2014).

47. Heathman, T. R. J. et al. Expansion, harvest and cryopreservation of human mesenchymal stem cells in a serum-free microcarrier process. Biotechnol. Bioeng. 112, 1696-1707 (2015).

48. Rafiq, Q. A., Brosnan, K. M., Coopman, K., Nienow, A. W. \& Hewitt, C. J. Culture of human mesenchymal stem cells on microcarriers in a 51 stirred-tank bioreactor. Biotechnol. Lett. 35, 1233-1245 (2013).

49. Petry, F. et al. Manufacturing of human umbilical cord mesenchymal stromal cells on microcarriers in a dynamic system for clinical use. Stem Cells Int. 2016, (2016).

50. Schirmaier, C. et al. Scale-up of adipose tissue-derived mesenchymal stem cell production in stirred single-use bioreactors under low-serum conditions. Eng. Life Sci. 14, 292-303 (2014).

51. Elseberg, C. L. et al. Microcarrier-based expansion process for hMSCs with high vitality and undifferentiated characteristics. Int. J. Artif. Organs 35, 93-107 (2012).

52. de Bournonville, S., Geris, L. \& Kerckhofs, G. Data from: MicroCT and contrast-enhanced microCT characterization of microcarriers in dry and wet state. https://doi.org/10.17632/rf6hsw3f2d.1 (2020).

53. Lee, S. J. \& Yang, S. Substrate curvature restricts spreading and induces differentiation of human mesenchymal stem cells. Biotechnol. J. 12, 1700360 (2017).

54. De Clercq, K. et al. High-resolution contrast-enhanced microCT reveals the true three-dimensional morphology of the murine placenta. Proc. Natl. Acad. Sci. U. S. A. 116, 13927-13936 (2019).

55. Kerckhofs, G. et al. Contrast-enhanced nanofocus X-ray computed tomography allows virtual three-dimensional histopathology and morphometric analysis of osteoarthritis in small animal models. Cartilage 5, 55-65 (2014).

56. Boulocher, C., Magnier, L., Roger, T., Viguier, E. \& Chereul, E. Preliminary study: non destructive evaluation of early cartilage morphological changes in a rabbit model by equilibrium partitioning of an ionic contrast agent by microcomputed tomography (EPIC- $\mu$ CT). Osteoarthr. Cartil. 20, S66 (2012).

57. Xie, L. et al. Magnetic resonance histology of age-related nephropathy in the Sprague Dawley rat. Toxicol. Pathol. 40, 764-778 (2012).

58. Otsu, N. Threshold selection method from gray-level histograms. IEEE Trans. Syst. Man Cybern. SMC-9, 62-66 (1979).

59. Meyer, F. Topographic distance and watershed lines. Signal Process. 38, 113-125 (1994).

60. Kirkpatrick, D. G. \& Seidel, R. On the shape of a set of points in the plane. IEEE Trans. Inf. Theory 29, 551-559 (1983).

61. Lehmann, G. \& Legland, D. Efficient N-Dimensional surface estimation using Crofton formula and run-length encoding. Insight J. January-De, 1-11 (2012).

\section{Acknowledgements}

S.d.B. was supported by a PhD grant of the Research Foundation Flanders (FWO, Grant No. 1S67217N, www.fwo. be). The CE-CT images have been generated at the X-ray computed tomography facilities of the Department of Development and Regeneration of the KU Leuven, financed by the Hercules Foundation (project AKUL 13/47). This work is part of Prometheus, the Leuven Research \& Development Division of Skeletal Tissue Engineering of the KU Leuven: www.kuleuven.be/prometheus.

\section{Author contributions}

S.d.B., L.G. and G.K. conceived the design of the experiments. S.d.B. performed the imaging of the microcarriers. S.d.B. designed and validated the image processing algorithms. Data interpretation was done by S.d.B., L.G. and G.K. S.d.B. and G.K. drafted the manuscript. All authors critically reviewed and approved the final manuscript.

\section{Competing interests}

The authors declare no competing interests.

\section{Additional information}

Supplementary Information The online version contains supplementary material available at https://doi. org/10.1038/s41598-021-81998-8.

Correspondence and requests for materials should be addressed to G.K.

Reprints and permissions information is available at www.nature.com/reprints.

Publisher's note Springer Nature remains neutral with regard to jurisdictional claims in published maps and institutional affiliations. 
(c) (i) Open Access This article is licensed under a Creative Commons Attribution 4.0 International cc) License, which permits use, sharing, adaptation, distribution and reproduction in any medium or format, as long as you give appropriate credit to the original author(s) and the source, provide a link to the Creative Commons licence, and indicate if changes were made. The images or other third party material in this article are included in the article's Creative Commons licence, unless indicated otherwise in a credit line to the material. If material is not included in the article's Creative Commons licence and your intended use is not permitted by statutory regulation or exceeds the permitted use, you will need to obtain permission directly from the copyright holder. To view a copy of this licence, visit http://creativecommons.org/licenses/by/4.0/.

(C) The Author(s) 2021 